\title{
Is Holmium Laser an Appropriate Modality to Treat Genital Warts?
}

\author{
Saleh Ghiasy', Morteza Fallah-Karkan', Mohammad Reza Razzaghi', Arash Ranjbar', Amirhosein Rahavian', \\ Babak Javanmard ${ }^{2 *}$
}

'Laser Application in Medical Science Research Center, Shahid Beheshti University of Medical Sciences, Tehran, Iran ${ }^{2}$ Department of Urology, Shohadae-Tajrish Hospital, Shahid Beheshti University of Medical Sciences, Tehran, Iran

*Correspondence to Babak Javanmard, MD; Assistant Professor of Urology, Shahid Beheshti University of Medical Sciences, Tehran, Iran. Tel: +982122749221; Email:

drbabakjavanmard@gmail.com

Published online 15 December 2018

\begin{abstract}
Introduction: Genital warts in young adults aged 18-28 years are very common. Several approaches are routinely used in the treatment of warts, viz., medical treatment (podophyllin and trichloroacetic acid), conventional surgery (excision or electrocautery), cryotherapy, and laser treatment. Because of high recurrence rates after treatment, complications and long duration of treatment, newer modalities have been developed. One of these newer methods is laser, which has been used in several urologic diseases. However, there are only a few studies about use of Holmium laser for treatment of genital warts. This retrospective study compared the success rate of Holmium laser with other available treatments for genital warts.

Methods: Between October 2011 and May 2016, 142 patients with genital warts attended the urology clinics at Shohada-e-Tajrish hospital in Tehran, Iran. Out of these, a total of 101 patients were included in this study consisting of 42, 39, 11 and 9 patients treated with cryotherapy, laser, conventional surgery and podophyllin respectively.

Results: The most successfully cleared lesions were seen in the holmium laser treatment group $(P=0.001)$. The lowest recurrence rate was observed in the holmium laser treatment group $(P=0.001) .17$ patients had one of these following problems: dysuria, initial hematuria or a change in the force and caliber of their urinary stream that after physical examination showed them to have a meatal wart. These patients then underwent cystoscopy up to urinary sphincter. All of them in addition to the meatus wart had a penile shaft lesion(s). Thirteen patients had meatal lesions, 9 of whom received holmium laser therapy and 4 patients were treated with electrocautery. Based on routine follow up after treatment, none of the patients treated with holmium laser had urinary stricture, but one case treated with electrocautery returned with a penile urethral stricture.

Conclusion: This study showed that treatment with Holmium laser has the highest clearance rate $(92.2 \%)$ and lowest recurrence rate $(14.3 \%)$ compared to other available treatments in this study. It may be concluded that holmium laser is a safe and effective treatment for genital warts with a low rate of recurrence.

Keywords: Human papilloma viruses (HPVs); Genital warts; Holmium laser; Cryotherapy; Conventional surgery; Podophyllin.
\end{abstract}

\begin{abstract}
Introduction
Human papilloma viruses(HPVs) have extended spectrum genotypes that include nearly 120 types involving the skin and mucosa of genitourinary tract, additionally it has approximately 40 distinct subtypes that infect anogenital tract. ${ }^{1}$ The most common and low-risk types of HPV are 6 and 11 and the most dangerous of HPV types are 16 and 18 which are considered high-risk types. ${ }^{2,3}$ In men, HPV is associated with anogenital cancers, specifically of the anus and penis. Approximately $90 \%$ of anal cancers are associated with HPV and of those with HPV, 90\% are due to HPV 16 and 18 . The most important route of virus transmission is sexual contact. ${ }^{4}$
\end{abstract}

The incidence of wart in young adults of the age group of 18-28 years is high. Data show that approximately 3-4 million cases of genital warts are occurred each year in men with a peak rate of 500 per 100000 in 25-29 years old group. ${ }^{5}$ Genital warts lead to increased economic burden and decreased quality of life. In actual fact the there is no curative treatment for HPV infection. ${ }^{6}$

Several approaches are routinely used for the treatment of wart, viz., medical treatment (podophyllin and trichloroacetic acid), conventional surgery (excision or electrocautery), cryotherapy and laser treatment. Cryotherapy and electrosurgery have been found to be more effective than other treatments, but none of these 
treatments are very successful. $^{7}$

Because of high recurrence rate after treatment, complications and a long duration of treatment, new modalities have been developed. One of these newer methods is the laser, which has been used in several urologic diseases. ${ }^{8-10}$

One type of laser is the holmium laser that is used for several urologic diseases. Mechanism of this advanced technology is photothermal destruction, that makes it possible to remove even the largest warts. ${ }^{11}$ However, there are few studies about the use of holmium laser in the treatment of genital warts. This retrospective study compared the success rate of holmium laser with other genital wart treatments.

\section{Methods}

Between October 2011 and May 2016, 142 patients presented with genital warts to the Urology Clinics in Shohda-e-Tajrish hospital in Tehran, Iran. The inclusion criteria were every patient referred to the Urology Clinics and had complete data with at least 6 months regular follow-up. In 31 cases the data was incomplete and nine cases that underwent medical treatment with trichloroacetic acid were excluded from this study. Finally, a total of 101 patients included for this study consisted of 42, 39, 11 and 9 patients treated with cryotherapy, laser, conventional surgery and podophyllin respectively.

Patients underwent one of 4 treatments: (1) cryotherapy with nitrous oxide using a Spembly (Andover, Hants) series PCG $12 \mathrm{R}$ cryoprobe with no local anaesthetic, which produced ice-balls 1-2 mm larger than the diameter of the wart (no electrolyte or lubricant was used). After warts were removed, optical tetracycline ointment was rubbed on the surface for 24 hours. (2) Holmium laser (manufactured in Iran) therapy with following parameters: Fiber caliber $200 \mathrm{Mm}$, maximum energy $0.8-1.0 \mathrm{~J}$, pulse frequency $8 \sim 12 \mathrm{~Hz}$, average power $6 \sim 12$ W. After routine decontamination of the lesions' area in the laser therapy group, the wart was treated by holmium laser with $1 \mathrm{~mm}$ margin of normal skin, by insertion of the laser fiber into the surface of warts. After warts were destroyed, residual necrotic tissue was cleaned with gauze (Figure 1). (3) The patients who underwent conventional surgery were treated by excision or electrocautery, or a combination of these methods. (4) Twenty-five percent podophyllin in tincture of benzoin compound was supplied by a local pharmacy. With care taken to avoid painting the surrounding skin, podophyllin was applied to the wart with a cotton swab. Patients were instructed to wash off the podophyllin 2 hours after the first treatment; this interval was lengthened by 2 hours to a maximum of 12 hours with each successive treatment.

Based on available data, 48 patients followed up for 6 months, 36 patients for 8 months and 17 patients for 2 years.
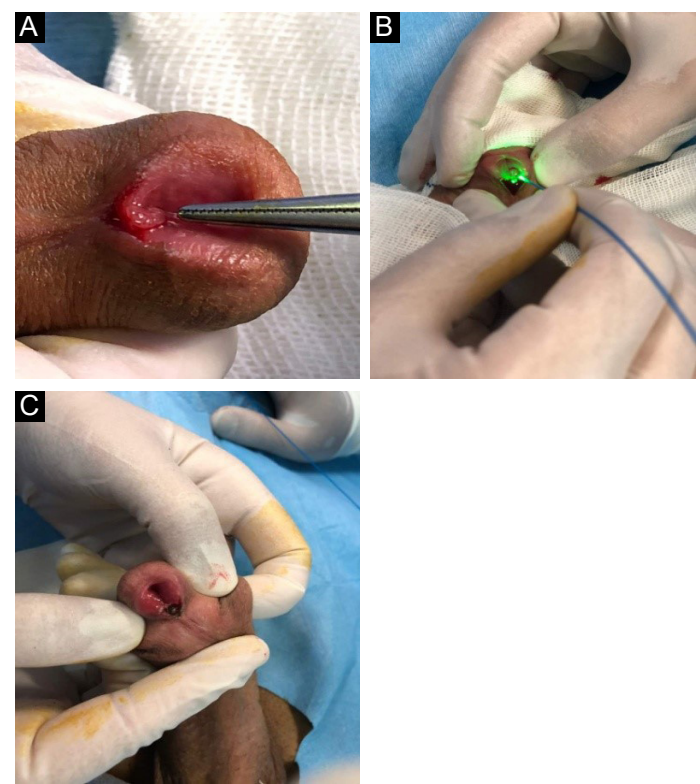

Figure 1. Lesion Treated by Holmium.

Patients' information was collected from medical records. Patients were followed 1, 6 and 16 weeks after primary treatment based on routine follow-up protocols. Effectiveness of treatment was calculated from recorded data as follows: (1) Clearance of lesions (number of cleared lesions divided by total number of lesions), and (2) Recurrence rate.

\section{Statistical Analysis}

Statistical analysis was performed using a commercially available software package (SPSS Statistics 17.0.0, SPSS, Chicago, Illinois). One-sample Kolmogorov-Smirnov test was utilized to estimate whether data was normally distributed. Parametric and nonparametric analyses were performed based on the finding of this evaluation. A $P$ value of 0.05 or less was considered statistically significant in this study.

\section{Results}

In the study a total of 101 men were included with mean age of 31.2 (standard deviation [SD] of \pm 9 ) (range: 16-49 years). The marital status of patients were single (67.4\%), married $(24.7 \%)$, divorced $(7.9 \%)$.

Patients had varied number of lesions ranged from 1 to 31 (median: 9) that were located on the penile shaft + scrotum $(27.7 \%)$, penile shaft alone $(24.7 \%)$, penile shaft + urinary meatus (19.8\%), scrotum (9.9\%), scrotum + perineum $(6.9 \%)$, glans $(5.9 \%)$, glans + scrotum $(2.9 \%)$ and penile shaft + perineum $(1.9 \%)$.

Thirty-three patients (35.6\%) had unprotected sex and one of them who had undergone conventional surgery was HIV positive. Twenty-two patients had history of previous genital wart.

The maximum number of cleared lesions were seen in 
Table 1. Result of Different Genital Wart Treatments

\begin{tabular}{|c|c|c|c|c|c|c|}
\hline & Total & Cryotherapy & Holmium Laser & Conventional Surgery & Podophyllin & $P$ Value \\
\hline No. of patients & 101 & 42 & 39 & 11 & 9 & \\
\hline Age $($ mean $\pm \mathrm{SD})$ & $31.2 \pm 9$ & $32.1 \pm 8.7$ & $32.5 \pm 9.1$ & $26 \pm 3.3$ & $27.8 \pm 12.4$ & 0.09 \\
\hline No. of lesions (mean \pm SD) & $9.8 \pm 5.7$ & $8.6 \pm 4.3$ & $10.7 \pm 6.2$ & $9 \pm 4.5$ & $13 \pm 9.3$ & 0.1 \\
\hline Clearance of treated lesions & $6 \pm 4.9$ & $3.6 \pm 1.8$ & $9.9 \pm 5.7$ & $3.3 \pm 1.6$ & $3.8 \pm 2.7$ & 0.001 \\
\hline No. of lesions recurrence( $\sim)$ & $4.1 \pm 2.6$ & $2.8 \pm 1.4$ & $1.5 \pm 0.8$ & $3 \pm 1.5$ & $9.3 \pm 6.7$ & 0.001 \\
\hline \multicolumn{7}{|c|}{ No. of lesions recurrence according to the F/U Time } \\
\hline Week 1( ) & $0.17 \pm .13$ & 0 & 0 & 0 & $0.7 \pm .53$ & \\
\hline Week 6( ) & $0.9 \pm .77$ & $0.1 \pm .05$ & $0.28 \pm .15$ & $0.11 \pm .58$ & $3.25 \pm 2.3$ & \\
\hline Week 16( ) & $3 \pm 1.68$ & $2.7 \pm 1.35$ & $1.22 \pm .65$ & $2.89 \pm .92$ & $5.3 \pm 3.8$ & \\
\hline
\end{tabular}

the holmium laser treatment group $(P=0.001)$ (Table 1$)$. Clearance in cryotherapy group, need for second- and third-time treatment sessions for eradication in $10 \%$ and $11.2 \%$ of lesions, respectively, while in the holmium laser and Conventional surgery groups, the clearance of all the lesions was noted after the first treatment.

The lowest recurrence rate was observed in the holmium laser group $(P=0.001)$ while the most recurrence rate was observed in the Podophyllin group (Table 1).

Seventeen patients had one of these following chief complaints: dysuria, initial hematuria or a change in the force and caliber of their urinary stream that after physical examination showed as having a meatal wart. These patients underwent cystoscopy up to the urinary sphincter. All of them in addition to the meatal wart had penis shaft lesion.

Thirteen patients had meatal lesions and 9 of them received holmium laser therapy (Figure 1A-C) and 4 patients were treated with electrocautery. One patient underwent meatotomy and wart excision and another patient because of multiple lesions, became candidate for marsupialization then buccal mucosa graft onlay surgery after 6 months. Based on routine follow-up after treatment, none of the patients treated with holmium laser had urinary stricture but one case treated with electrocautery returned with penile urethral stricture.

\section{Discussion}

Genital wart treatments divided into 2 categories one that is applied directly to the skin such as medical treatment and one that needs surgery to remove warts and includes freezing with liquid nitrogen (cryotherapy), conventional surgery (excision or electrocautery) and laser treatment. ${ }^{12-14}$

Over the last decade there is a trend towards surgical treatment rather than radical medical treatment in terms of management of genital warts. The modality chosen is based on the number, size, and location of the warts. The benefits of surgical modalities include the following: increased clearance rates and decreased pain, lower recurrence rate and lesser side effects. Today, laser and cryotherapy are considered the most successful treatments.

Medical treatment of warts with podophyllin has reported different rates of clearance between 22 to $79 \% .^{15-18}$ Treatment with podophyllin may be associated with contact dermatitis. ${ }^{19}$

Conventional surgeries include excision or electrocautery require anesthesia. Treatment mechanism of electrocautery is thermal injury, and in surgical excision the lesion is removed with scalpel. Studies showed that clearance rate of electrocautery may be as efficient as cryotherapy. Surgical excision can eradicate lesions with low risk of recurrence. ${ }^{20}$

Cryotherapy is one of the destructive methods for genital warts with acceptable clearance rates and no harmful systemic side effects. ${ }^{21}$ It is an effective treatment for those warts which were hard to treat with other modalities and there is no need for anesthesia. It has not become popularly accepted because of some limitations including unusual techniques and equipment. ${ }^{22}$

Treatment with lasers is one of the recommended management options for warts, especially in cases which are refractory, thick and extensive lesions. ${ }^{23}$ In comparison with other treatments, usage of laser needs local anesthesia and is accompanied by scar itchiness, but destroys lesions more radically. ${ }^{21}$ The benefits of laser include the following: painless, quick healing, lower infection levels and limited rate of recurrence rates and complications. holmium laser has been used widely in urologic surgery but there are few studies reported about usage of holmium laser in genital warts. ${ }^{8}$

In our study, $92.2 \%$ of lesions were cleared with holmium laser in first session while $41.8 \%$ of patients showed clearance of warts after 3 sessions of treatment during 2 weeks in cryotherapy group. The cure rate of conventional surgery was $37.1 \%$ and analysis show $29.8 \%$ of cases treated with podophyllin obtained perfect clearance 
within 4-6 weeks of treatment.

Previous trials have reported a cure rate of $60 \%-100 \%$, $60 \%-97 \%, 56 \%-93.3 \%$ and $19 \%-80 \%$ when genital warts were treated with holmium laser, cryotherapy, conventional surgery and podophyllin respectively. ${ }^{20,24,25}$ Treatment with podophyllin and cryotherapy are simpler procedures than other treatments while holmium laser needs a laser machine and fibre.

In terms of recurrence rate, this study shows a recurrence rate of $14.3 \%, 33.4 \%, 34.2$ and $71.8 \%$ when lesions removed by holmium laser, cryotherapy, conventional surgery and podophyllin respectively.

Previous studies showed recurrence rates of 3\%-77\%, $20 \%-79 \%, 8 \%-35 \%$ and $23 \%-70 \%$ when lesion treatment had done with holmium laser, cryotherapy, conventional surgery and podophyllin respectively. $711,16,20$

The results of this study showed treatment with holmium laser is correlated with highest clearance rate (92.2\%) and lowest recurrence rate $(14.3 \%)$ than other applied treatments. In conclusion, holmium laser is safe, and more effective, than other methods in management of genital warts.

\section{Limitation and Recommendation}

Limitations of this study include its retrospective nature, short-term follow-up, and lack of information about genotyping of HPV.

It is recommended that more studies should be conducted as randomized clinical trial with long-term follow-up to determine the rate of recurrence. In addition, the effects of laser therapy on female genital wart are required.

\section{Ethical Considerations}

The Ethical Committee of Shohada- e-Tajrish hospital approved this study and permitted us to review patients' medical data.

\section{Conflict of Interests}

The authors declare no conflict of interest.

\section{Acknowledgments}

We thank the Shohada-e-Tajrish Hospital medical records staff who helped using data collection.

\section{References}

1. Azizjalali M, Ghaffarpour G, Mousavifard B. CO2 Laser therapy versus cryotherapy in treatment of genital warts; a Randomized Controlled Trial (RCT). Iran J Microbiol. 2012;4(4):187.

2. Parkin DM, Bray F. The burden of HPV-related cancers. Vaccine. 2006;24:S11-S25. doi:10.1016/j. vaccine.2006.05.111

3. de Villiers E-M, Fauquet C, Broker TR, Bernard H-U, zur Hausen H. Classification of papillomaviruses. Virology. 2004;324(1):17-27. doi:10.1016/j.virol.2004.03.033

4. Daling J, Sherman K. Relationship between human papillomavirus infection and tumours of anogenital sites other than the cervix. IARC Sci Publ. 1992;(119):223-41.

5. Insinga RP, Dasbach EJ, Myers ER. The health and economic burden of genital warts in a set of private health plans in the United States. Clin Infect Dis. 2003;36(11):1397-1403. doi:10.1086/375074

6. Hallaji Z, Noroozi-Nejad E. Genital warts in 250 Iranian patients and their high-risk sexual behaviors. Arch Iran Med. 2013;16(9):518.

7. Stone K, Becker T, Hadgu A, Kraus S. Treatment of external genital warts: a randomised clinical trial comparing podophyllin, cryotherapy, and electrodesiccation. Genitourin Med. 1990;66(1):16-9.

8. Razzaghi MR, Fallahkarkan M, Ghiasy S, Javanmard B. Laser application in Iran urology: a narrative review. J Lasers Med Sci. 2017;9(1):1-6. doi:10.15171/jlms.2018.01

9. Fallah Karkan M, Ghiasy S, Ranjbar A, Javanmard B. Evaluation of $200 \mathrm{Mm}, 365 \mathrm{Mm}$ and $500 \mathrm{Mm}$ fibers of Ho:YAG laser in transurethral lithotripsy of ureteral: a randomize control trial. J Lasers Med Sci. 2018;9(1):6972. doi:10.15171/jlms.2018.14

10. Javanmard B, Fallah Karkan M, Razzaghi MR, Ghiasy S, Ranjbar A, Rahavian A. Surgical management of vesical stones in children: a comparison between open cystolithotomy, percutaneous cystolithotomy and transurethral cystolithotripsy with holmium-yag laser. J Lasers Med Sci. 2018;9(3):183-187. doi:10.15171/ jlms.2018.33

11. Yang C-J, Liu S-X, Liu J-B, et al. Holmium laser treatment of genital warts: an observational study of 1500 cases. Acta Derm Venereol. 2008;88(2):136-8. doi: 10.2340/00015555-0354

12. Sonnex C, Lacey CJ. The treatment of human papillomavirus lesions of the lower genital tract. Best Pract Res Clin Obstet Gynaecol. 2001;15(5):801-816. doi:10.1053/beog.2001.0221

13. Ting PT, Dytoc MT. Therapy of external anogenital warts and molluscum contagiosum: a literature review. Dermatol Ther. 2004;17(1):68-101.

14. Maw R. Critical appraisal of commonly used treatment for genital warts. International Journal of STD \& AIDS. 2004;15(6):357-64. doi:10.1258/095646204774195173

15. Workowski KA, Bolan GA; Centers for Disease Control and Prevention. Sexually transmitted diseases treatment guidelines. MMWR Recomm Rep. 2015;64(RR-03):1-137.

16. Douglas JM Jr, Eron LJ, Judson FN, et al. A randomized trial of combination therapy with intralesional interferon $\alpha 2 b$ and podophyllin versus podophyllin alone for the therapy of anogenital warts. J Infect Dis. 1990;162(1):529. doi:10.1093/infdis/162.1.52

17. Potkul R, Lancaster W, Kurman R, Lewandowski G, Weck P, Delgado G. Vulvar condylomas and squamous vestibular micropapilloma. Differences in appearance and response to treatment. J Reprod Med. 1990;35(11):1019-22.

18. A comparison of interferon alfa- $2 \mathrm{a}$ and podophyllin in the treatment of primary condylomata acuminata. Genitourin Med. 1991;67:394-9.

19. Tabari ST, Javadian M, Barat S. The efficacy of podophylin $20 \%$ and thricholoroacetic acid $30 \%$ in the treatment of 
genital wart. Casp J Intern Med. 2010;1(1):16-9.

20. Beutner KR, Wiley DJ, Douglas JM, et al. Genital warts and their treatment. Clin Infect Dis. 1999;28(Suppl 1):S37-S56. doi:10.1086/514722

21. Scheinfeld N, Lehman DS. An evidence-based review of medical and surgical treatments of genital warts. Dermatol Online J. 2006;12(3):5.

22. Balsdon M. Cryosurgery of genital warts. Br J Vener Dis. 1978;54(5):352.

23. Workowski KA, Berman S. Sexually transmitted diseases treatment guidelines, 2010. MMWR Recomm Rep. 2010 Dec 17;59(RR-12):1-110.

24. McMillan A, Scott G. Outpatient treatment of perianal warts by scissor excision. Sexually Transmitted Infections. 1987;63(2):114-5.

25. Jensen SL. Comparison of podophyllin application with simple surgical excision in clearance and recurrence of perianal condylomata acuminata. Lancet. 1985;326(8465):1146-1148. 